\title{
Plasma membrane localization of paralogous leucine permeases Bap2 and Bap3 is regulated by Bul1
}

\author{
Maheswaran. $S^{1}$ and Paike Jayadeva Bhat ${ }^{1}$ \\ ${ }^{1}$ Department of Biosciences and Bioengineering, Indian Institute of Technology, Bombay. \\ Mumbai. India. \\ Correspondence to maheshking.un@gmail.com
}

\begin{abstract}
Timeliness in expression and degradation of the nutrient permeases is crucial for any organism. In Saccharomyces cerevisiae, post translational regulation of nutrient permeases such as trafficking and turnover are poorly understood. We found that loss of a leucine permease BAP2, but not other permeases lead to severe growth retardation when the carbon source is glucose or galactose but not glycerol and lactate. Leucine prototrophy suppressed the retardation, showing $B A P 2$ and $L E U 2$ are synthetically lethal. We discovered that loss of $B U L 1$, an arrestin involved in trafficking of diverse permeases suppressed this lethality. The suppression required another leucine permease, $B A P 3$. Our results suggest that $B U L 1$ downregulate permeases $B A P 2$ and $B A P 3$ present in plasma membrane through Rsp5 dependent endocytosis. We speculate that by regulating leucine import $B U L 1$ regulates the activity of TORC1.
\end{abstract}

\section{Introduction}

Leucine is a unique amino acid. It is the highest occurring amino acid in polypeptides and also functions as a signal (Crozier et al., 2005; Stipanuk, 2007; Suryawan et al., 2011; Ananieva et al., 2016). Target of Rapamycin (TOR) the mastermind of cell growth and proliferation in eukaryotes is activated by leucine (Bonfils et al., 2012; Han et al., 2012; Gonzalez and Hall, 2017). Leucine consumption has implications in several diseases. Leucine deprivation results in stalling of breast cancer growth and induces apoptosis (Xiao et al., 2016). Leucine and its breakdown products reduce the glucose uptake, in adult cardiomyocytes even after insulin treatment by affecting translocation of GLUT4, a glucose transporter, establishing a possible link between diabetes and leucine consumption (Renguet et al., 2017).

Despite its ability to make all 20 proteinogenic amino acids, S.cerevisiae has amino acid permeases to import amino acids from the external milieu (Ljungdahl and Daigan-Fornier, 2012). These permeases are synthesized in rough endoplasmic reticulum, forwarded to golgi bodies from where they are either dispatched to plasma membrane or vacuole for degradation (Lauwers et al., 2009). When not needed, as in case of absence of substrates, to limit the import in case of excess substrates or while experiencing stress like deviation from optimal temperature, $\mathrm{pH}$, heavy metal ions, improper folding permeases are removed from the plasma membrane through endocytosis, sent to vacuole for degradation (Abe and Iida, 2003; Kim et al., 2007; Lin et al., 
2008; Nikko et al., 2008; Nikko and Pelham, 2009; Hatakeyama et al., 2010; O’Donnell et al., 2010; Becuwe et al., 2012a; Zhao et al., 2013; Suzuki et al., 2013; Ghaddar et al., 2014; Crapeau et al., 2014; Talaia et al., 2017; Hovsepian et al., 2017; Hovsepian et al., 2018; Savocco et al., 2019; Tanahashi et al., 2021).

Prior to endocytosis, permeases are ubiquitylated by Rsp5 Ubiquitin ligase with the help of adaptor proteins known as $\underline{\text { Arrestin }}$ Related Trafficking adaptors (ARTs) also called simply as arrestins (Lin et al., 2008; Belgareh-Touze et al., 2008; Leon and Tsapis., 2009). Although many ubiquitin ligases are known, only the HECT domain Rsp5 ligase, is so far reported to participate in permease ubiquitylation. There are about 10-12 cytoplasmic arrestins and 5-6 membrane bound arrestins in S.cerevisiae (Nikko and Pelham, 2009). Each arrestin is responsible for ubiquitylation of a set of permeases and conversely a permease shall have one or more arrestins to facilitate ubiquitylation (O'Donnell and Schmidt, 2019). After ubiquitylation, the permease is endocytosed to form an early endosome, which matures to a late endosome before finally fusing with the vacuole (Rotin et al., 2000; Hettema et al., 2004). This turnover and insertion of newly synthesized permeases in the plasma membrane is necessary for the cell to cope and survive in a constantly changing environment.

Here, we show that the growth of S.cerevisiae is critically dependent on BAP2, a high affinity leucine permease, either in a synthetic medium containing several amino acids or in a minimal medium with scanty leucine. This observation comes as a surprise considering the ability of several other permeases to transport leucine. We observed that loss of BUL1, an arrestin, overcame the dependency on $B A P 2$, by stabilizing $B A P 3$ in the plasma membrane. We show that the turnover of leucine permeases Bap2 and Bap3 is accomplished by Bul1. Finally, we propose that by regulating the abundance of these permeases, Bul1 modulates the activity of TORC1, which in turn regulates Bul1, thus setting up a feedback loop. Hence, this study for the first time provides a possible mechanism as how leucine regulates its own uptake. 


\section{Results}

BAP2 and LEU2 are synthetically lethal in carbon source dependent manner

Commonly used wild type strains (WT) of BY4741, W303 and $\sum 1278 \mathrm{~b}$ background are leucine auxotrophs (leu2A) and suffer a growth defect in a modified SCGlu medium ( Synthetic Complete Glucose medium) (Cohen and Engelberg, 2007). The growth defect is due to impaired leucine uptake, suppressed by multicopy plasmids either bearing LEU2, BAP2 or TAT1 (Cohen and Engelberg, 2007). We believe that the impairment is due to a fierce competition between leucine and other surplus competing amino acids for their import through a common transporter(s). In such a case, reduced abundance of the competing amino acids would favor leucine import and restore growth. Likewise, WT BY4741 grows well in the routine SCGlu medium, which has only 12 amino acids in lower concentrations (Fig.1A), opposed to all 20 amino acids in high concentrations in the modified SCGlu medium.

The failure of the strains to import leucine in modified SCGlu medium was intriguing, in view of the presence of several leucine permeases such as Bap2, Bap3, Tat1, Tat2, Agp1, Gnp1 etc. We sought to ascertain the importance of these permeases in growth. We studied the growth of strains lacking leucine permeases and found that loss of BAP2 could establish the leucine import deficient growth defect in the routine SCGlu medium (Fig.1A). The growth of bap2 $\Delta$ strain was restored by LEU2 complementation (Fig.1B). Among other permeases loss of TAT1 conferred a mild growth defect. Interestingly, the growth defect vanished when the carbon source was swapped to glycerol and lactate (SCGlylac-Synthetic Complete Glycerol and lactate medium) but not galactose (SCGal-Synthetic Complete Galactose medium) (Fig.1C).

We report for the first time that $B A P 2$ and $L E U 2$ are synthetically lethal in SCGlu/Gal medium. But why would loss of $B A P 2$, confer growth retardation when other leucine permeases are also expressed in SCGlu medium (Forsberg et al., 2001)? Perhaps, BAP2 is the predominant leucine transporter and its loss amounts to growth retardation.

\section{Growth defect of bap2 2 strain is overcome by loss of BUL1}

We wondered if the growth defect be overcome by ways other than complementation by LEU2. In a tryptophan import deficient mutant, loss of $B U L 1$, an arrestin restores import by stabilizing TAT2, a permease which imports Tryptophan, Tyrosine, Phenylalanine and Leucine etc. in plasma membrane (Umebayashi and Nakano, 2003). Actually, loss of BUL1, in the bap2A strain did restore growth normalcy in SCGlu and SCGal media (Fig.2A). But the growth of bap $2 \triangle$ bul1 1 strain was not dependent on TAT2, GNP1 or AGP1, but BAP3 (Fig.S1A,B). We speculated that loss of $B U L 1$, led to $B A P 3$ accumulation in plasma membrane.

As expected Bap3 accumulation was enriched in the periphery of the bap2 $\Delta$ bul1 $\Delta$ strain than the bap2 $\triangle$ strain (Fig.2B). Contrastingly, vacuolar Bap3 distribution was enriched in a bap $2 \Delta$ strain than the bap $2 \Delta$ bul1 1 strain. This pattern of Bap3 distribution was more prominent 
while comparing bull $\triangle$ and WT strains (Fig.2C). This observation shows that irrespective of the presence or absence $B A P 2$, Bul1 downregulates Bap3. When blotted, membranous Bap3-EGFP population was enriched in bullA strain than the WT (Fig.2D).

These results show that loss of $B U L 1$, results in $B A P 3$ accumulation in cell membrane. Going by its canonical role, we surmise that $B U L 1$ is responsible for downregulation of BAP3 in plasma membrane through Rsp5 mediated endocytosis. Unlike SCGlu medium, we did not observe any discernible difference in Bap3 distribution between bap2 $\triangle$ and bap2 4 bul1 1 or WT and bullA strains grown in SCGal medium (Fig.2E,F).

\section{Loss of BUL1 empowers WT to grow in an extremely low leucine environment}

We wondered how the bap2 $\triangle$ strain would grow in a medium devoid of amino acids other than auxotrophic needs. Hence we chose SD medium ( Synthetic Minimal Dextrose) for this study (Fig.3A). The bap2 $\triangle$ strain grew well as WT in SD75 medium (75 $\mathrm{M}$ leucine), but ceased to grow at SD25 medium $(25 \mu \mathrm{M}$ leucine). The growth was restored through loss of $B U L 1$, and required $B A P 3$. At SD5 medium ( $5 \mu \mathrm{M}$ leucine), even WT ceased to grow. Once again, loss of $B U L 1$ restored WT its growth which required $B A P 2$ not $B A P 3$.

These results show that $B A P 2$ and $L E U 2$ are synthetic lethal when (1) surplus of competing amino acids are present (SCGlu/Gal) or (2) leucine is scarce (SD). For the bap2 $\triangle$ strain at SD75, absence of competing amino acids favors seamless leucine import through other permeases. This holds true for a bap2 4 bap3 3 strain also. But in SD25, other permeases do not sustain the growth of bap2 $\triangle$ strain, unless Bap3 accumulates at plasma membrane through loss of $B U L 1$. In SD5, where leucine is very scarce, even WT doesn't grow unless BAP2 accumulates at plasma membrane due to loss of BUL1. We surmise that Bul1 downregulates both Bap2 and Bap3 at plasma membrane.

The bul1 4 strain displayed slightly increased in plasma membrane Bap3 localization, than the WT (Fig.3B). Though visible difference in vacuolar distribution was not observed. Unlike Bap3, Bap2 was enriched in plasma membrane of the bul14 strain than the WT and the vacuolar distribution was quite opposite (Fig.3C). For Fig.3C, we used SD25 broth as growing of cells in SD broth was extremely difficult. Immunoblotting results showed a larger Bap2-yeGFP and lesser free GFP population in the bulld strain than the WT (Fig.3D). Our attempts to perform immunoblotting of Bap3-EGFP was not quite successful probably due to much reduced expression of Bap3-EGFP. These results indicate that loss of BUL1, leads to accumulation of Bap2 and Bap3 in plasma membrane possibly due to reduced downregulation. 


\section{$B A P 2$ is the major leucine transporter in SCGlu medium}

As we suppose $B A P 2$ as the major leucine transporter in SCGlu medium, we tried to know how much leucine does BAP2 import, how loss of BUL1 affects the import in bap2A strain? etc. We measured the import of C14-L-Leucine in SCGlu medium for strains as in (Fig.4A). As strains like bap2A doesn't grow in SCGlu medium, all the strains were made prototrophic for leucine to facilitate their growth. We assumed that the leucine prototrophy doesn't affect the import by the permeases and their regulation by BUL1.

Assuming WT import capacity as $100 \%$, bap $3 \Delta$, bap $2 \Delta$ and bap $2 \Delta$ bap $3 \Delta$ strains displayed $10 \%, 40 \%$ and $40 \%$ drop respectively. This implied BAP2 as the major and BAP3 as a minor leucine transporters in SCGlu medium. In bap2 $\triangle$ strain, loss of BUL1 doubled its import, which vanished on loss of BAP3. Interestingly, loss of BUL1 in the bap3A strain augmented its import by $50 \%$. This was due to apparent accumulation of $B A P 2$ at the plasma membrane, as loss of $B A P 2$ not only eliminated this effect but also reduced its import alike to that of a bap $2 \triangle$ strain. Loss of $B U L 1$, in WT increased the import by $30 \%$, possibly due to plasma membrane accumulation of both $B A P 2$ and BAP3. Import data for bap $2 \triangle$ and bap3 $\triangle$ strains agree with earlier reports (Grauslund et al., 1995; Didion et al., 1998).

\section{Loss of BUL2 does not suppress the growth defect of bap2 $\Delta$ strain}

$B U L 2$ and $B U L 1$ are paralogues, together they downregulate many permeases (Yashiroda et al., 1998; O'Donnell and Schmidt, 2019). WT strain used in this study is naturally bul2 $\Delta$ as it harbors a loss of function allele of $B U L 2\left(B U L 2^{F 883 L}\right.$ ) (Kwan et al., 2011). Perhaps the suppression of the bap $2 \Delta$ growth defect through accumulation of Bap3 at plasma membrane is due to the collective loss of $B U L 1$ and $B U L 2$. In such case, we suppose that the expression of a

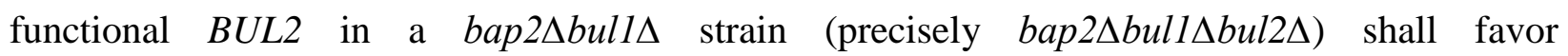
downregulation of Bap3 and confer back the growth defect at least partially if not fully.

To check this idea, the bap2 $\Delta$ bul1 $\Delta$ strain was complemented with a functional BUL2 allele from W303-1A strain background alongside BUL1 as a positive control (Fig.S2). But it was not BUL2 but BUL1 to confer the growth defect back. We believe that BUL2 does not downregulate Bap3 at plasma membrane and the suppression is solely due to loss of BUL1 but not BUL2. The functionality of the BUL2 allele was verified as it successfully restored the growth of the bul1 $\Delta$ strain (precisely bul1 $\Delta b u l 2 \Delta$ ) which suffered a severe growth defect at $37^{\circ} \mathrm{C}$ in SMTGlu (Synthetic Minimal Tyrosine Glucose medium) (Fig.S3). 


\section{Discussion}

Organisms auxotrophic for nutrients rely on import from extracellular medium. Though Saccharomyces cerevisiae is naturally prototrophic for all amino acids, lab strains are usually auxotrophic for afew amino acids. Strains auxotrophic for leucine, tyrosine and tryptophan have difficulty in growth due to their poor import of corresponding amino acids from the media with surplus amino acids (Cohen and Engelberg, 2007).

In this study, in an otherwise normally growing WT strain (leu2 $\triangle$ ), loss of BAP2 confers a growth defect due to poor import of leucine. The growth defect was suppressed by accumulation of Bap3 at plasma membrane due to loss of an arrestin, BUL1. Also in a $B A P 2^{+}$ strain, loss of $B U L 1$ leads to plasma membrane accumulation of Bap2. With these results we propose Bul1 as a post translational regulator to regulate the abundance of Bap3 and Bap2 at plasma membrane possibly through ubiquitin conjugated endocytosis. This finding joins the long list of transporters regulated by Bul1.

SPS (Ssy1-Ptr3-Ssy5) is an amino acid sensor present in plasma membrane. SSY1 or PTR3 and LEU2 are synthetically lethal in SCGlu medium, overcome by loss of BUL1 (Forsberg et al., 2001). This study finds that the pair BAP2 and LEU2 alone is sufficient to confer synthetic lethality in SCGlu and other media too. In presence of extracellular amino acids, SPS amino acid sensor prompts transcription of permeases AGP1, GNP1, BAP2, BAP3, TAT1 and TAT2, all of which can import leucine at varying rates (Forsberg et al., 2001). We suppose $B A P 2$ as the major importer of leucine as loss of $B A P 2$ alone causes the growth defect and $40 \%$ drop in import. Galactose and glycerol upsurge leucine import by tenfold than glucose (Peter et al., 2006), yet the growth defect persists in SCGal not in SCGlylac medium. Again it seems that BAP2 is the major leucine importer in SCGal, while in SCGlylac other leucine permeases account for the hike. These observations oblige to revisit the amino acid metabolism/uptake in terms of the Carbon source, instead the Nitrogen source or amino acids as it has been customarily.

We speculate a signaling loop whereby leucine regulates its import (Fig.4B). Leucine binds Cdc60, a leucyl tRNA synthetase cum intracellular leucine sensor to activate TORC1, via EGO complex bound Rag GTPases Gtr1 and Gtr2 (Bonfils et al., 2012). Active TORC1 releases Bul1 from Npr1 kinase inhibition (Merhi and Andre, 2012). Bul1 promotes Bap2 and Bap3 endocytosis, causing drop in leucine import and intracellular levels, eventually reducing TORC1 activity.

Loss of BUL2 reduces chronological lifespan (CLS) and prolongs replicative lifespan (RLS), due to suspected increased TORC1 activity, as a result of increased intracellular amino acid pool (Kwan et al., 2011). Then how the loss of BUL1 would affect the lifespans? With our observations and fact that Leucine activates TORC1, we expect BUL1 to have a greater bearing than BUL2. Contradicting the above, CLS of a leu2A strain is extended by leucine prototrophy or increasing leucine concentration in the medium (Alvers et al., 2009). 
Being the most sought amino acid for translation, a slight shortage of leucine may harshly affect the growth. Adhering to this notion bap2 $\Delta$, bap2 $\Delta$ bap3 $\Delta$ and bap2 $\Delta$ bap3 3 bull $\Delta$ strains that show $40 \%$ reduction in leucine import are the ones to suffer growth defect (Fig.4C). We think of two reasons to explain this scenario. The intracellular leucine concentration is below the threshold (1) to bind Cdc60 and activate TORC1. As a result growth ceases when TORC1 is not active. (2) to charge Cdc60 for translation. 


\section{Main figures}

\section{Fig.1.}

A

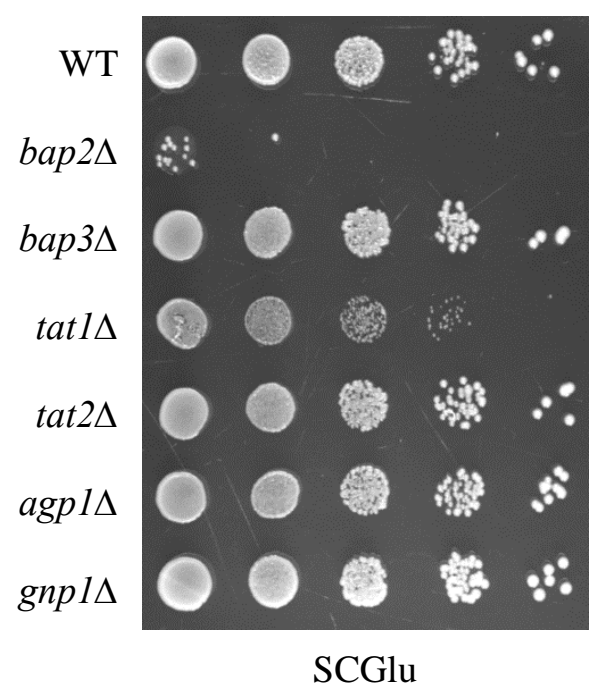

C

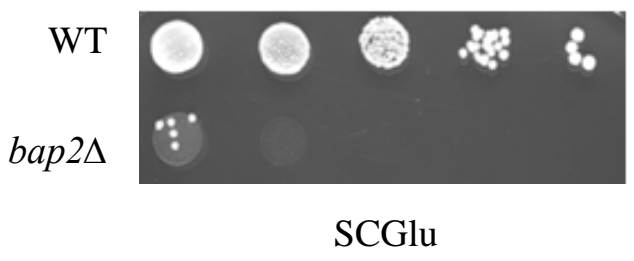

WT

bap $2 \Delta$



SCGal

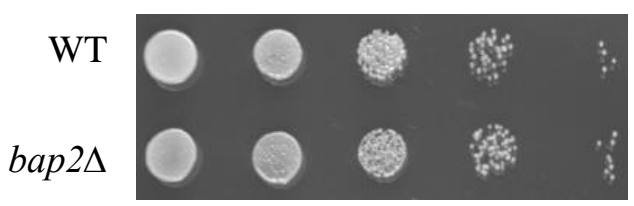

SCGlylac
B

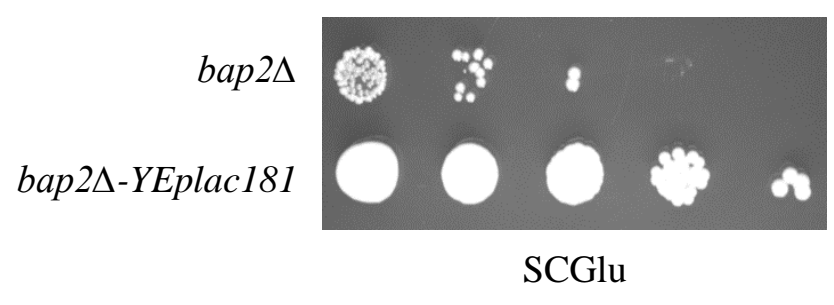


Figure 1. BAP2 and $L E U 2$ are synthetically lethal in a carbon source dependent manner. (A) WT, bap2 2 , bap3 3 , tat $1 \Delta$, tat $2 \Delta$, agp $1 \Delta$ and gnp $1 \Delta$ strains grown in SCGlylac broth were spotted on SCGlu medium. (B) bap $2 \Delta$ strain and its transformant carrying YEplac181, a LEU2 plasmid grown in SCGlylac or SLeu Glylac broth were spotted on SCGlu medium. (C) WT and bap2 2 strains grown in SCGlylac broth were spotted on SCGlu, SCGal and SCGlylac media. Images were captured and analyzed after incubating the petri plates for $2-3$ days at $30^{\circ} \mathrm{C}$.

\section{Fig.2.}

$\mathbf{A}$

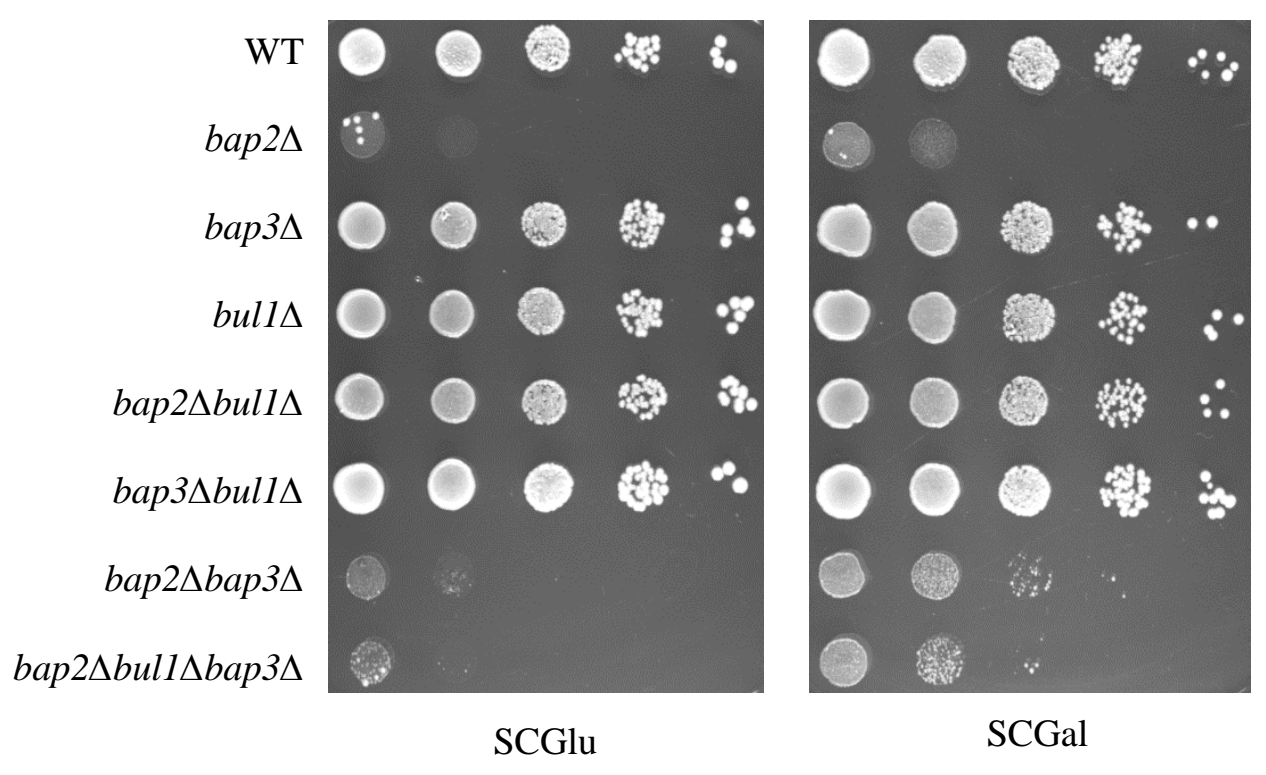

B

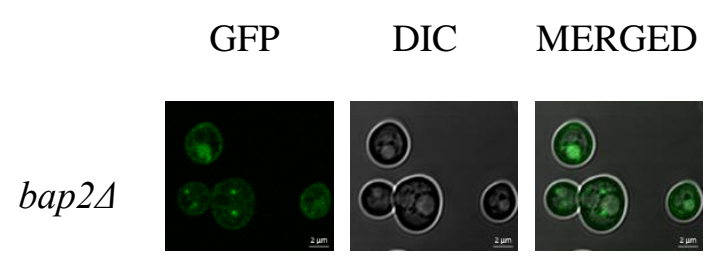

bap2 2 bul1
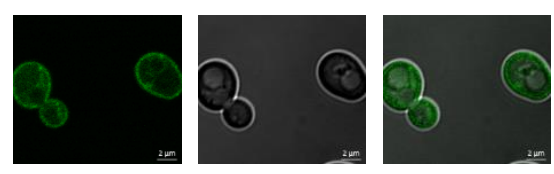
C

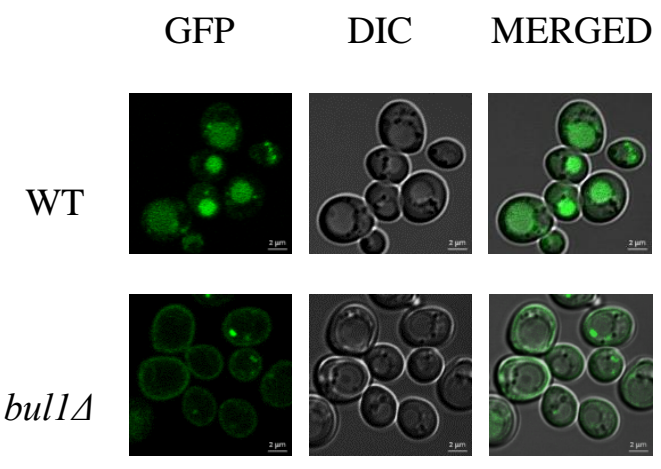

D

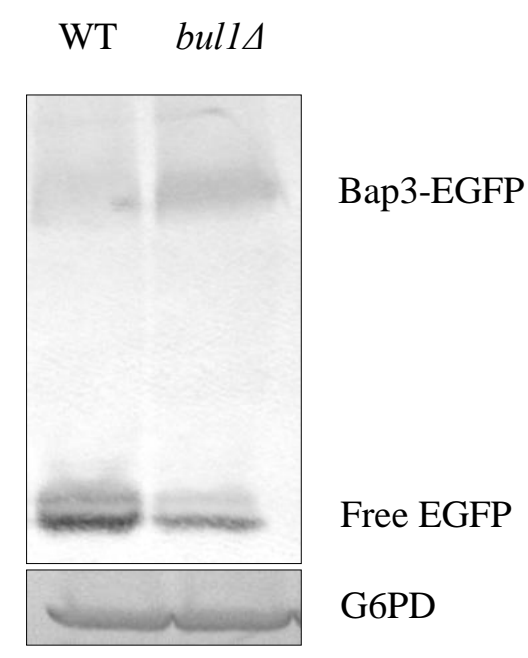

E

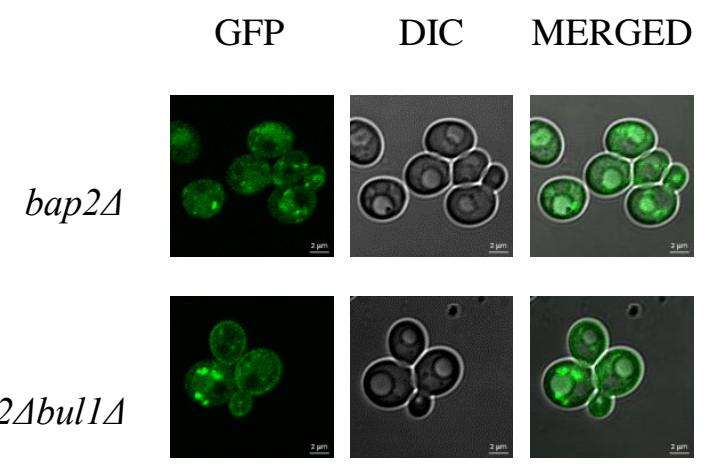




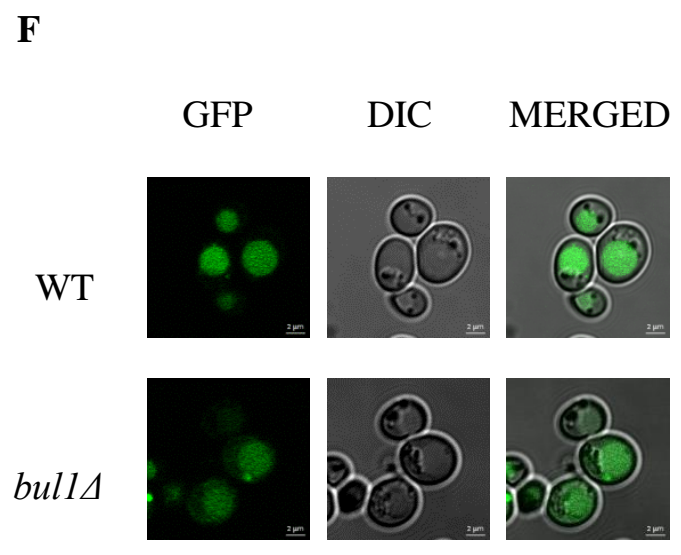

Figure 2. Loss of BUL1 suppresses bap2 $2 \Delta$ growth defect, by stabilizing Bap3 in the plasma


bap $2 \Delta$ bul1 $\Delta$ bap $3 \Delta$ strains grown in SCGlylac broth were spotted on SCGlu and SCGal media. Images were captured and analyzed after incubating the petri plates for $2-3$ days at $30^{\circ} \mathrm{C}$. (B) Localization of endogenously EGFP tagged BAP3 in bap $2 \Delta$ and bap2 $\triangle$ bull $1 \Delta$ strains grown in SCGlylac broth and subcultured in SCGlu broth upto mid logarithmic phase. Scale- $2 \mu \mathrm{M}$. (C) Localization of endogenously EGFP tagged BAP3 in WT and bul1 $\mathrm{strains}$ grown in SCGlylac broth and subcultured in SCGlu broth upto mid logarithmic phase. Scale-2 $\mu$ M. (D) Immunoblotting of total cell lysates of endogenously EGFP tagged BAP3 in WT and bull $1 \Delta$ strains, grown as mentioned in C. Anti-GFP and anti-G6PD antibodies (for loading control) were used for immunoblotting. Free EGFP emanates from vacuolar degradation of Bap3-EGFP chimera. (E) Localization of endogenously EGFP tagged BAP3 in bap2 $\Delta$ and bap $2 \Delta$ bul1 $\Delta$ strains grown in SCGlylac broth and subcultured in SCGal broth upto mid logarithmic phase. Scale- $2 \mu \mathrm{M}$. (F) Localization of endogenously EGFP tagged BAP3 in WT and bul1 $\triangle$ strains grown in SCGlylac broth and subcultured in SCGal broth upto mid logarithmic phase. Scale- $2 \mu \mathrm{M}$. 
Fig.3.

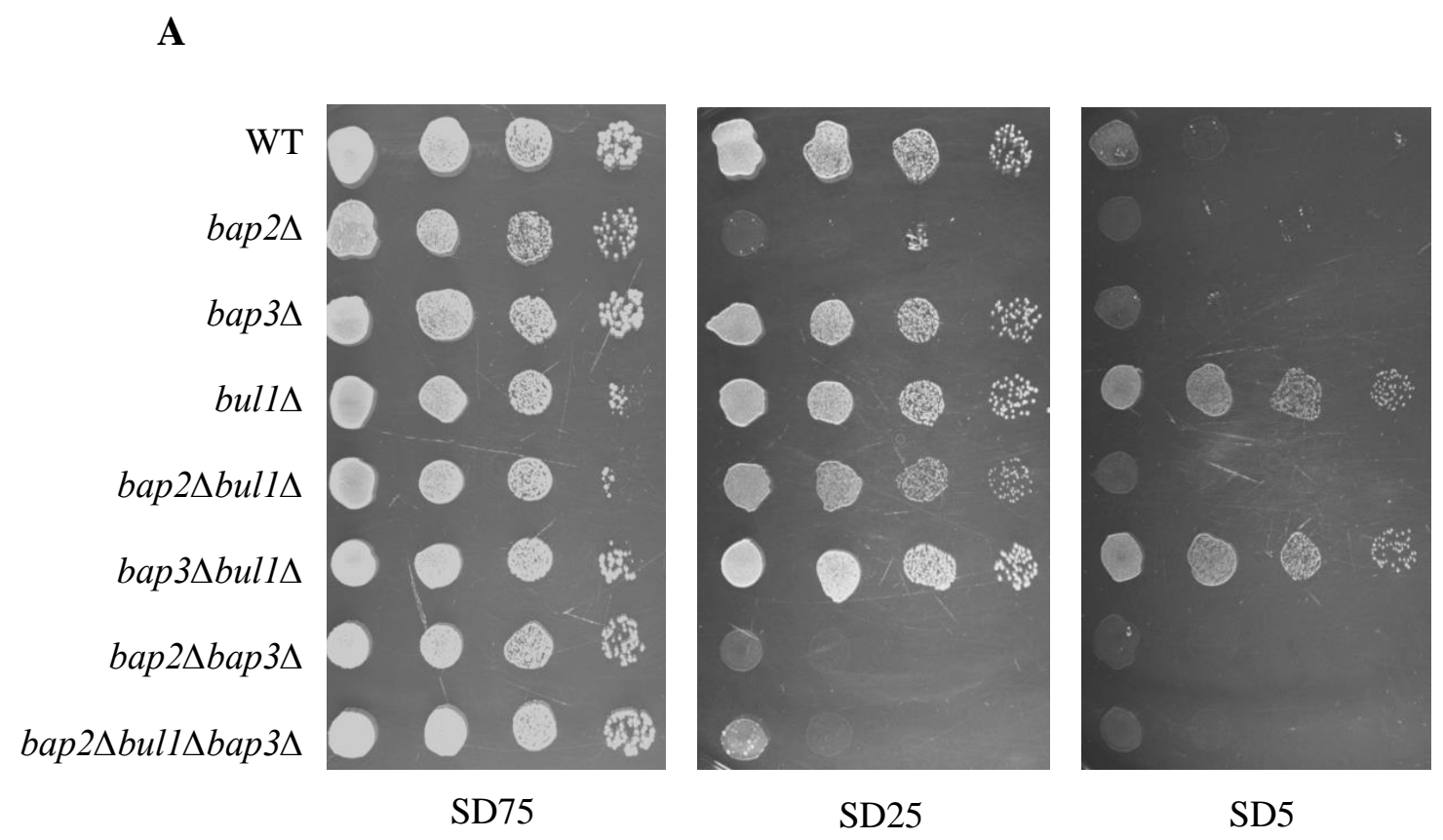

B

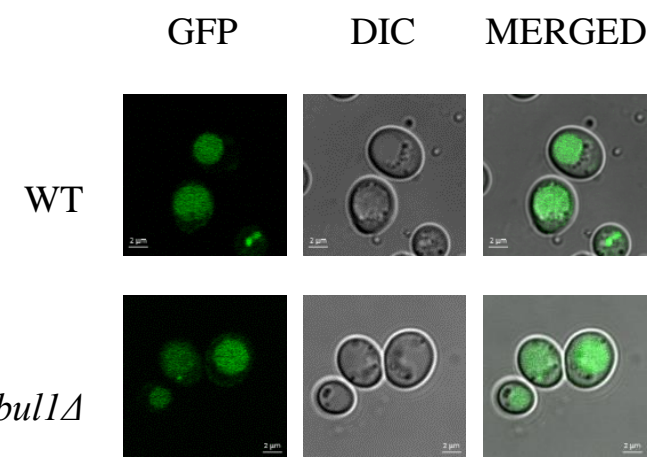

C

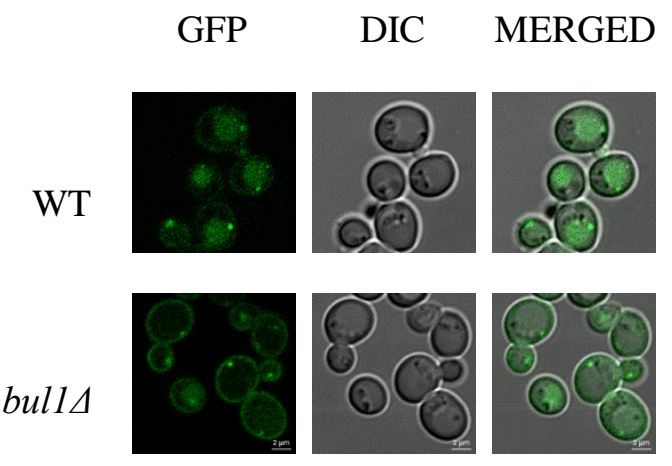


D
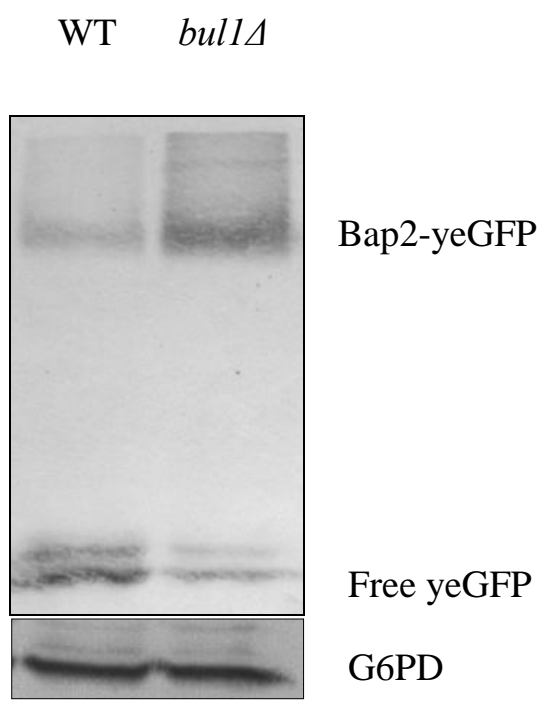

Figure 3. Loss of $B U L 1$ enables WT to grow in a medium with scarce leucine, through stabilization of Bap2 in the plasma membrane. (A) WT, bap $2 \Delta$, bap $3 \Delta$, bul1 $\Delta$, bap $2 \Delta$ bull $\Delta$, bap $3 \Delta$ bull $\Delta$, bap $2 \Delta$ bap $3 \Delta$ and bap $2 \Delta$ bull 1 bap $3 \Delta$ strains grown in SCGlylac broth were spotted on SD75, SD25 and SD5 media. Images were captured and analyzed after incubating the petri plates for $2-3$ days at $30^{\circ} \mathrm{C}$. (B) Localization of endogenously EGFP tagged BAP3 in WT and bul1 $\triangle$ strains grown in SCGlylac broth and subcultured in SD25 broth upto mid logarithmic phase. Scale- $2 \mu \mathrm{M}$. (C) Localization of endogenously yeGFP tagged BAP2 in WT and bul1 $\triangle$ strains grown in SCGlylac broth and subcultured in SD25 broth upto mid logarithmic phase. Scale-2 $\mu \mathrm{M}$. (D) Immunoblotting of total cell lysates of endogenously yeGFP tagged BAP2 in WT and bull 1 strains, grown as mentioned in C. Anti-GFP and anti-G6PD antibodies (for loading control) were used for immunoblotting. Free EGFP emanates from vacuolar degradation of Bap2-yeGFP chimera. 
Fig.4.

A

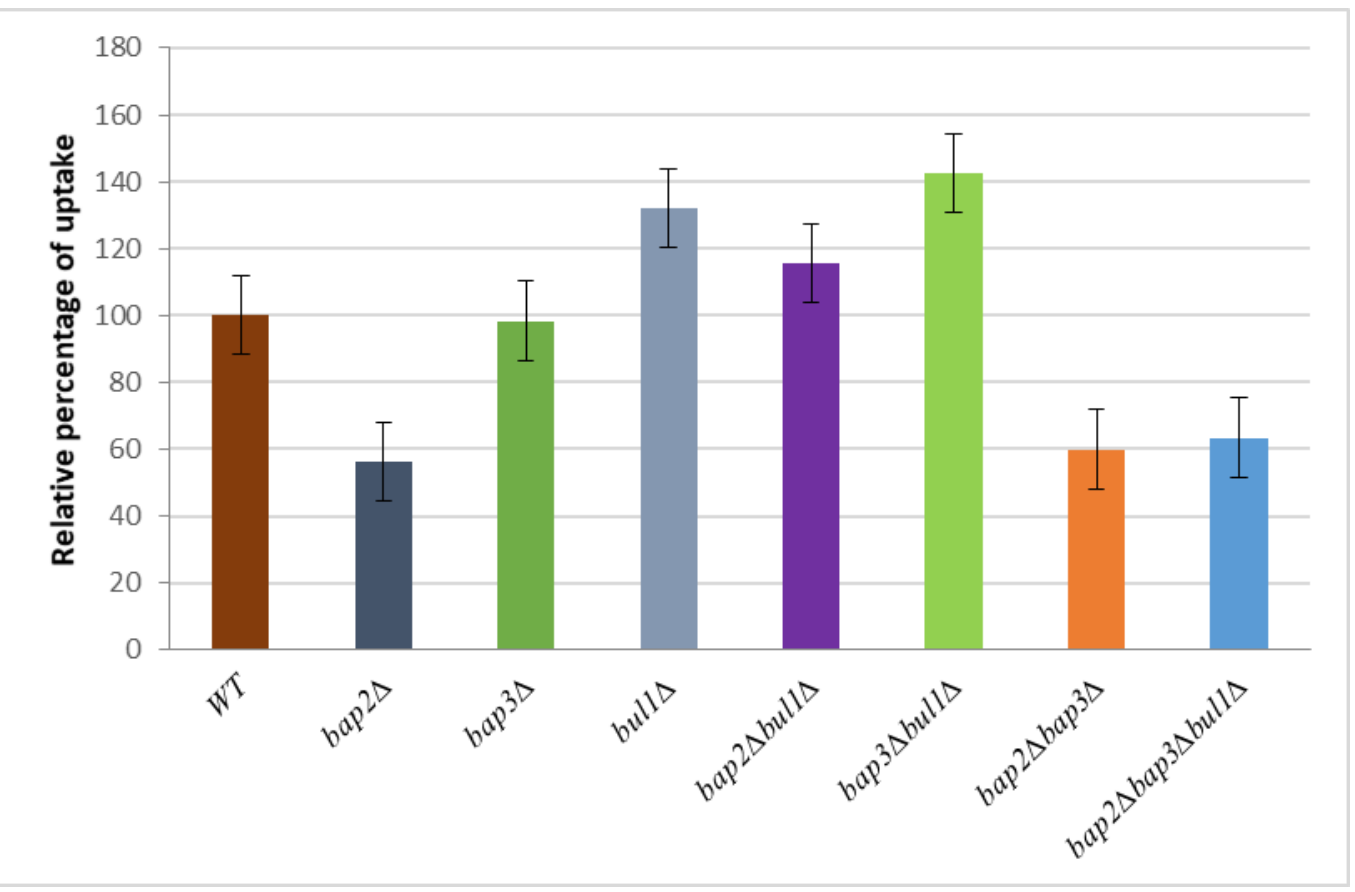

B




C

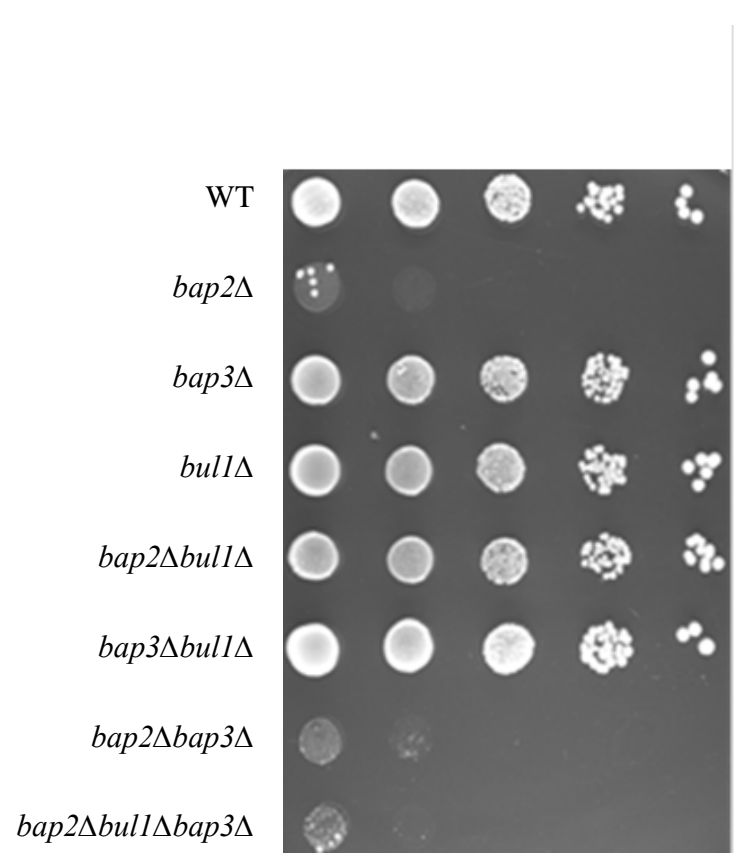

SCGlu

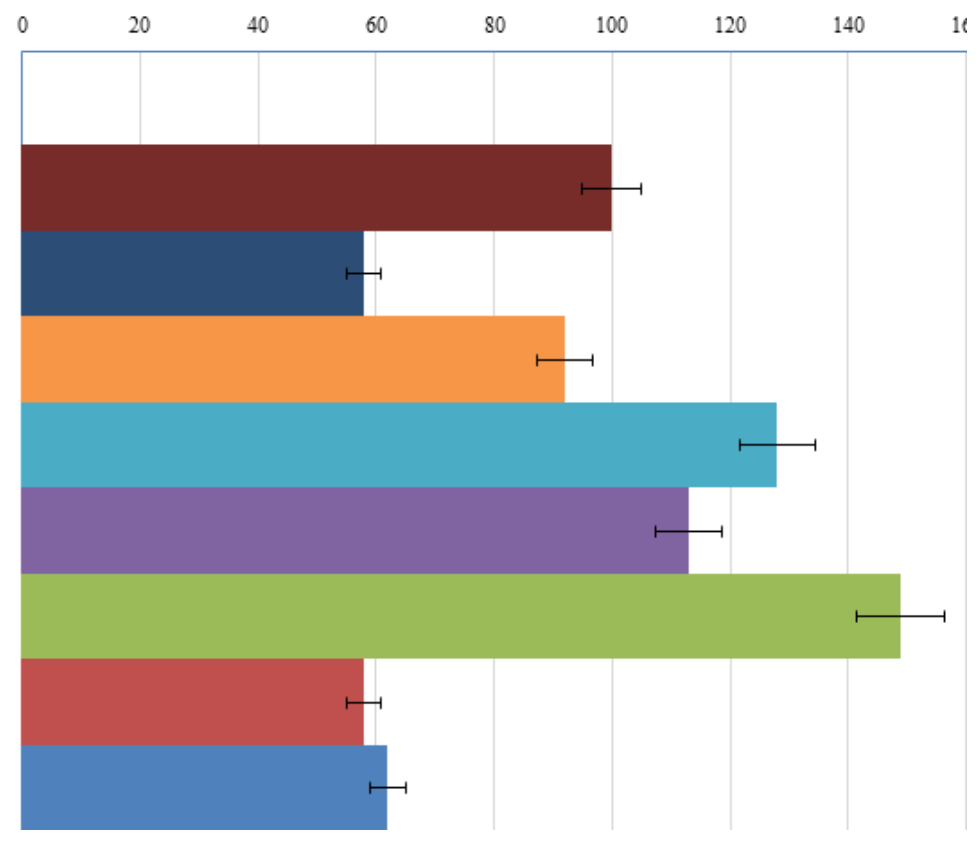

C14-Leucine uptake

Figure 4. Leucine availability is crucial for growth. (A) WT, bap $2 \Delta$, bap $3 \Delta$, bul1 1 , bap $2 \Delta$ bul1 $1 \Delta$, bap $3 \Delta$ bul1 $\Delta$, bap $2 \Delta$ bap $3 \Delta$ and bap $2 \Delta$ bul1 1 bap $3 \Delta$ transformants carrying YEplac181, a LEU2 plasmid were assayed for the uptake of $\mathrm{C} 14$ labelled L-Leucine. Uptake was calculated as picomoles $/ 0.8 \times 10^{6} \mathrm{cells} / \mathrm{min}$ and expressed as mean relative percentage $\pm \mathrm{SE}$, considering uptake of WT as $100 \%$. (B) Schematic representation of the proposed TORC1-Leucine feedback loop representing how leucine regulates TORC1 activity. (C) Correlation of growth phenotype of strains grown on SCGlu medium as shown in Fig.2A and their relative leucine uptake as shown in Fig.4A. 


\section{Supplementary figures}

\section{Fig.S1.}

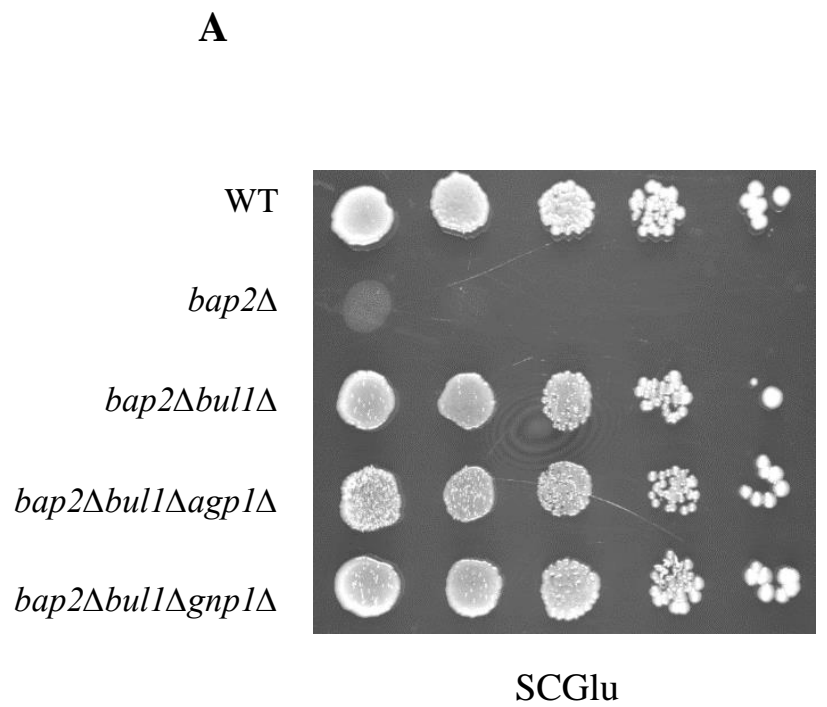

B

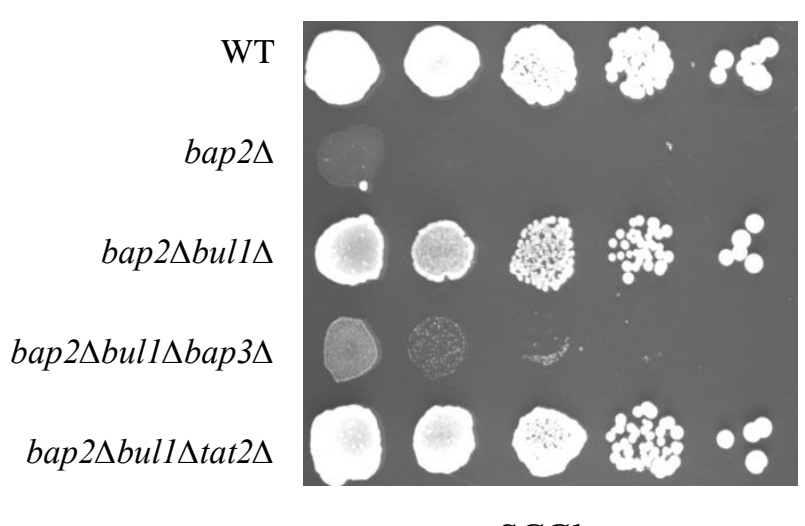

SCGlu

Figure S1. Loss of BUL1 suppresses the bap2 $\triangle$ growth defect, in a BAP3 dependent manner. (A) WT, bap $2 \Delta$, bap $2 \Delta$ bul1 $\Delta$, bap $2 \Delta$ bul1 $\Delta a g p 1 \Delta$ and bap $2 \Delta$ bul1 1 gnp $1 \Delta$ strains grown in SCGlylac broth were spotted on SCGlu medium. (B) WT, bap $2 \Delta$, bap $2 \Delta$ bul $1 \Delta$, bap $2 \Delta$ bull $1 \Delta$ bap $3 \Delta$ and bap $2 \Delta$ bul $1 \Delta$ tat $2 \Delta$ strains grown in SCGlylac broth were spotted on SCGlu medium. Images were captured and analyzed after incubating the petri plates for $2-3$ days at $30^{\circ} \mathrm{C}$. 


\section{Fig.S2.}



Figure S2. Complementation by BUL1 not BUL2 confers the growth defect back in the bap2 $\triangle$ bul1 $\Delta$ strain. WT, bap $2 \Delta$, bap $2 \Delta$ bul $1 \Delta$ strains and bap $2 \Delta$ bul $1 \Delta$ transformants carrying YCplac33 or YCplac33 with BUL1 or BUL2 grown in SCGlylac or SUra-Glylac broth were spotted for growth on SCGlu medium. Images were captured and analyzed after incubating the petri plates for $2-3$ days at $30^{\circ} \mathrm{C}$.

\section{Fig.S3.}

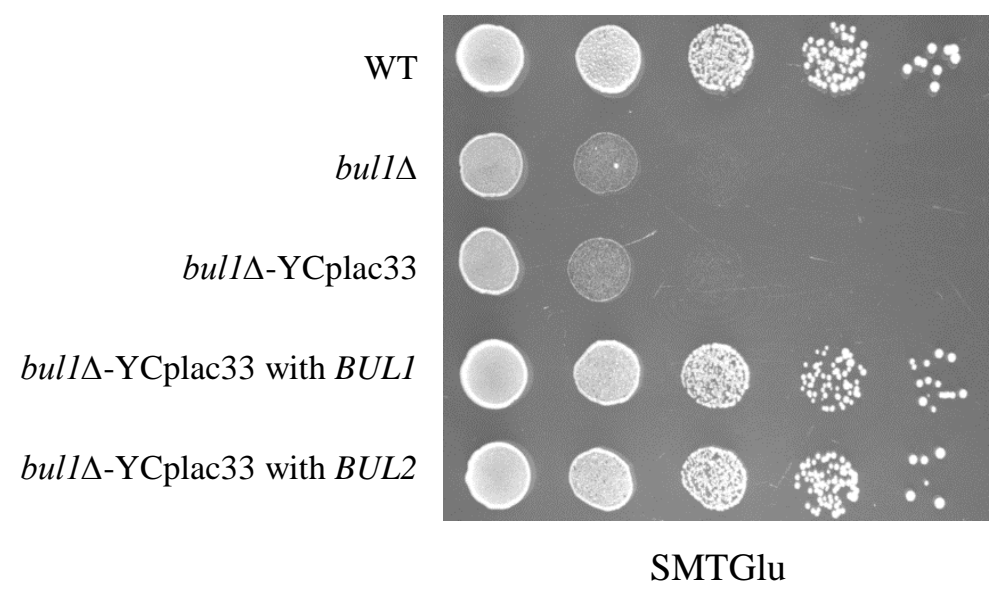


YCplac33 or YCplac33 with BUL1/BUL2 grown in SCGlylac or SUra-Glylac broth were spotted for growth on SMTGlu medium. Images were captured and analyzed after incubating the petri plates for 2-3 days at $37^{\circ} \mathrm{C}$. 


\section{Materials and methods}

\section{Yeast strains and plasmids}

All yeast strains are of BY4741 background (ST.1). They were either purchased from Euroscarf or constructed. Yeast transformation was performed by LiAc-PEG method. Yeast genomic DNA was isolated by glass bead method. Genetic manipulations like disruption and tagging were performed by PCR based homologous recombination method. These manipulations were again confirmed by PCR. Primers used for the above procedures are listed (ST.3).

E.coli strain XL1 or DH5 $\alpha$ were used for plasmid cloning and propagation. Plasmid isolation was done by alkaline lysis method and E.Coli transformation was done by PEG method. Plasmids used in the study are listed (ST.2). Plasmid carrying BUL1 was constructed by cloning of 4020 bp SalI-EcoRI fragment of BUL1 gene (1000 bp upstream of start codon, 2931 bp CDS and 89 bp downsream of stop codon), into SalI-EcoRI site of YCplac33. BUL1 was amplified from the genomic DNA of WT BY4741. Plasmid carrying BUL2, was constructed by cloning of a 4019 bp SalI-SacI fragment of BUL2 (1000 bp upstream of start codon, 2763 bp CDS, 250 bp downsream of stop codon, 6 bp of primer region), amplified from genomic DNA of WT W3031A strain, into the SalI-SacI site of YCplac33.

Make - Primers (Sigma, BLOT solutions), Restriction enzymes, polymerases and ligases (Thermo scientific, NEB), PCR/Gel purification kits (Qiagen).

\section{Media recipe}

Synthetic Complete Glucose or Synthetic Complete Galactose (SCGlu/SCGal) - 0.17\% Yeast nitrogen base, $0.5 \%$ ammonium sulfate, $0.05 \%$ amino acid mixture (ST4) and 2\% glucose or galactose. Synthetic Complete Glycerol plus lactate (SCGlylac) - 0.17\% Yeast nitrogen base, $0.5 \%$ ammonium sulfate, $0.05 \%$ amino acid mixture (ST4) and 3\% (V/V) glycerol and 2\% (V/V) of 40\% lactate pH 5.7. Synthetic Minimal Dextrose (SD) - 0.17\% Yeast nitrogen base, $0.5 \%$ ammonium sulfate, $2 \%$ glucose and $0.01 \%$ of auxotrophic supplements Uracil $(89 \mu \mathrm{M})$, Histidine $(64 \mu \mathrm{M})$, Methionine $(67 \mu \mathrm{M})$ and Leucine $(76 \mu \mathrm{M})$. SD75, SD25 and SD5 refer to SD with $75 \mu \mathrm{M}$ or $25 \mu \mathrm{M}$ or $5 \mu \mathrm{M}$ leucine. For solid media $2 \%$ agar was used. SUra ${ }^{-G l y l a c}$ and $\mathrm{SLeu}^{-}$ Glylac refers to SCGlylac lacking Uracil or Leucine respectively. Synthetic Minimal Tyrosine 
Glucose (SMTGlu) - $0.17 \%$ Yeast nitrogen base, 2\% glucose, $0.01 \%$ of auxotrophic supplements and $2 \mathrm{mM}$ Tyrosine.

\section{Spotting assay (Growth assay)}

Cells were grown in SCGlylac broth (SCGlylac broth lacking uracil or leucine for transformants) to mid log phase, harvested and washed with sterile distilled water and cell density was normalized to $\mathrm{OD}_{600}$ 1.0. The normalized cell suspension was serially diluted to tenfold dilutions upto $10^{-4}$ dilution. $5 \mu \mathrm{l}$ from each dilution was spotted onto appropriate media and incubated at $30^{\circ} \mathrm{C}$ or $37^{\circ} \mathrm{C}$ for $2-3$ days. Images of the petriplates were captured by UVITEC, CAMBRIDGE gel documentation system of GeNei.

\section{Protein extraction and immunoblotting}

Protein was extracted by alkaline lysis method. Cells grown in suitable medium to mid $\log$ phase were harvested at $4^{\circ} \mathrm{C}$ and lysed with $0.5 \mathrm{ml}$ of lysis solution $(0.2 \mathrm{M} \mathrm{NaOH}, 0.2 \%$ mercaptoethanol) for $10 \mathrm{~min}$ on ice. Trichloroacetic acid was added to a final concentration of $5 \%$, and incubated for another $10 \mathrm{~min}$ on ice. Samples were centrifuged for $5 \mathrm{~min}$ at $13000 \mathrm{x} \mathrm{g}$ for 5 min. Pellets were resuspended in $35 \mu \mathrm{l}$ of dissociation buffer (4\% SDS, 0.1M Tris- $\mathrm{HCl} \mathrm{pH}$ 6.8, 4mM EDTA, 20\% glycerol, 2\% 2-mercaptoethanol, 0.02\% Bromophenol blue) and mixed with $15 \mu \mathrm{l}$ of $1 \mathrm{M}$ Tris base, and heated at $37^{\circ} \mathrm{C}$ for $10 \mathrm{~min}$.

Total cell free extracts were resolved by SDS-PAGE and transferred to 0.45 micron nitrocellulose membrane. Mouse bi-clonal anti-GFP antibodies were used to probe GFP tagged proteins and chased by secondary anti-mouse goat antibody conjugated with alkaline phosphatase. Rabbit anti-G6PD antibody was used to probe G6PD, and chased down by secondary anti-rabbit mouse antibody conjugated with alkaline phosphatase. Blots were developed and images were captured by UVITEC, CAMBRIDGE gel documentation system of GeNei and analyzed by UVITEC1D software.

Make - Mouse bi-clonal anti-GFP antibody (Roche Diagnostics, Mix of 13.1 and 7.1 monoclonal antibodies), Rabbit anti-G6PD antibody (Santacruz Biotechnology) and Secondary anti-rabbit mouse antibody (Santacruz Biotechnology). Nitrocellulose membrane (Amersham Biosciences). SDS-PAGE and blotting transfer apparatus (GeNei). 


\section{Fluorescence microscopy}

Cells were grown in appropriate media to mid log phase were harvested, washed in sterile distilled water and resuspended in the same (To avoid auto fluorescence due to nutrient broth). Cells were readily analyzed for GFP fluorescence at room temperature under Zeiss AxioObserver Z1 inverted motorized and computer-controlled laser scanning fluorescence microscope (Carl Zeiss, LSM 780) equipped with iPlan-Apochromat 100X oil immersion objective 1.4 NA and PMT detector camera. Argon+ laser was used to excite the fluorophore with excitation wavelength of $488 \mathrm{~nm}$ and emission wavelength was $526 \mathrm{~nm}$. Images were captured and later processed by Zen 2012 blue acquisition software from Zeiss.

\section{Leucine uptake assay}

Yeast cells were grown in SCGlylac broth lacking leucine; subcultured in SCGlu broth and grown upto mid log phase $\left(\mathrm{OD}_{600}\right.$ 1-1.5). Cells were harvested, washed with SCGlu broth twice and resuspended in the same to $0.4 \times 10^{7} \mathrm{cell} / \mathrm{s} / \mathrm{ml}$. To $200 \mu \mathrm{l}$ of this suspension added was $2.8 \mathrm{KBq}$ (500 picomoles) of C14-labelled leucine. The suspension contained $188 \mu \mathrm{M}$ of cold leucine and $2.5 \mu \mathrm{M}$ of $\mathrm{C} 14-L e u c i n e$. The cell suspensions were incubated in $30^{\circ} \mathrm{C}$ incubator cum shaker for 0 min, 60 min and 120 min time points. The C14-labelled leucine uptake was stopped by adding cold leucine of $100 \mu \mathrm{l}$ to a final leucine concentration of $12.6 \mathrm{mM}$. The cells from the suspension were collected on a 0.45 micron nylon membrane (Amersham Biosciences) by suction. Cells were washed with ice cold water for 3 times and the membrane was dried for 10 minutes. The membrane with cells was counted for radioactive pulses for 1min/sample in Perkin Elmer Liquid scintillation analyzer. For each repetition biological replicates were taken and technical triplicates were taken for each strain for each time point. C14-Leucine with specific activity of $5.5 \mathrm{GBq} / \mathrm{mmole}(150 \mathrm{mci} / \mathrm{mmole})$ was purchased from BRIT, India. 


\section{Supplementary tables.}

\section{ST1. Yeast strains.}

\begin{tabular}{|c|c|c|}
\hline Strain & Genotype & Source \\
\hline WT & 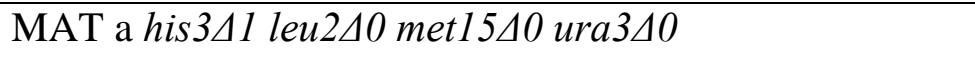 & Euroscarf \\
\hline bap24 & 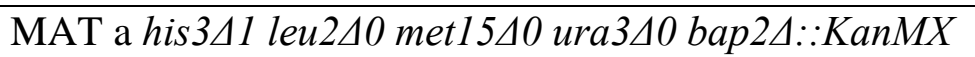 & This study \\
\hline bap34 & 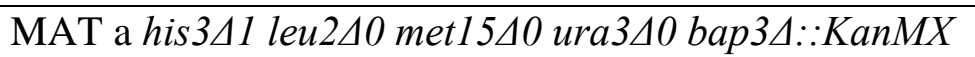 & This study \\
\hline tat1A & 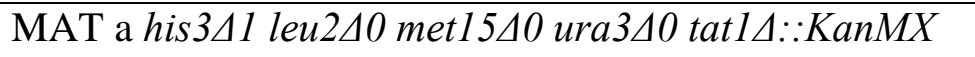 & This study \\
\hline tat2A & 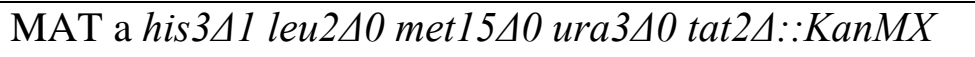 & This study \\
\hline agplA & 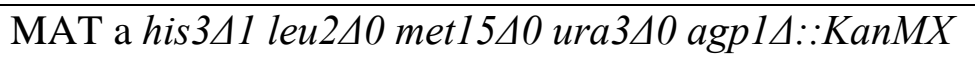 & This study \\
\hline gnp14 &  & This study \\
\hline bulld &  & This study \\
\hline bap2Abul1 & 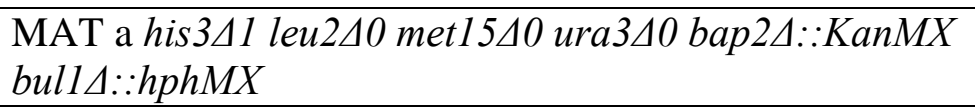 & This study \\
\hline bap3 3 bul1 1 & 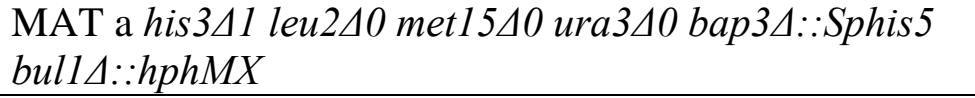 & This study \\
\hline bap2 2 bap3A & 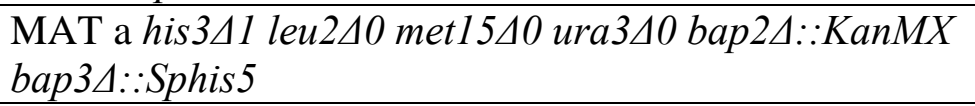 & This study \\
\hline bap2 2 bul1 $\triangle$ bap3A &  & This study \\
\hline 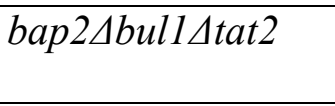 & 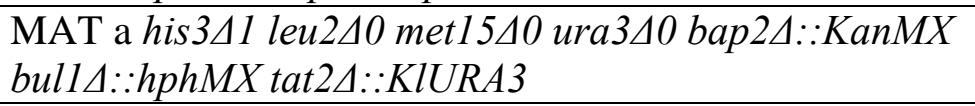 & This study \\
\hline 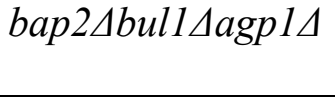 & 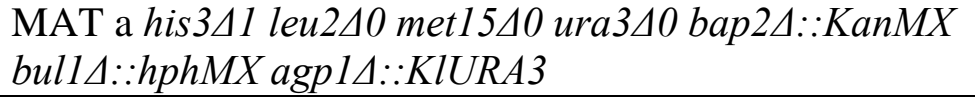 & This study \\
\hline  & 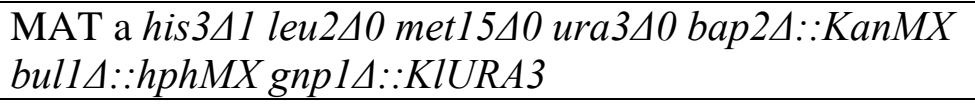 & This study \\
\hline $\begin{array}{l}\text { bap2 } \triangle B A P 3- \\
\text { EGFP }\end{array}$ & 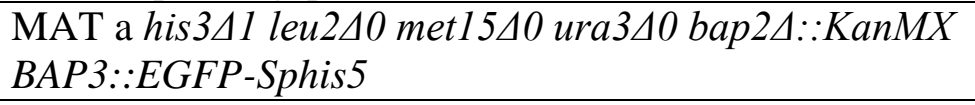 & This study \\
\hline $\begin{array}{l}\text { bap2 } \triangle \text { bul1 } \triangle B A P 3- \\
\text { EGFP }\end{array}$ & 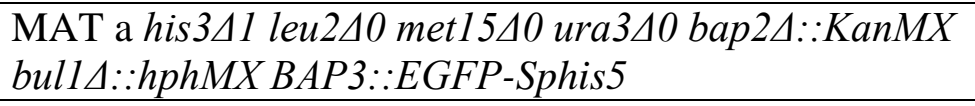 & This study \\
\hline BAP3-EGFP & 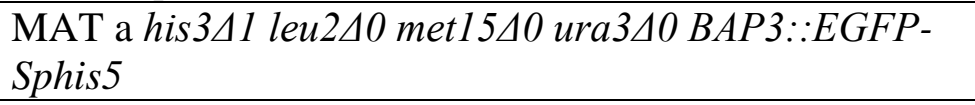 & This study \\
\hline bul1 $\triangle B A P 3-E G F P$ & 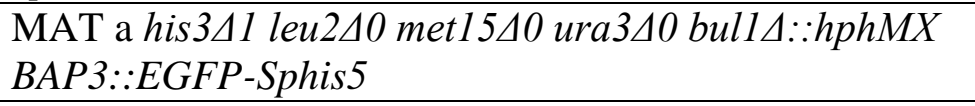 & This study \\
\hline BAP2-yeGFP & 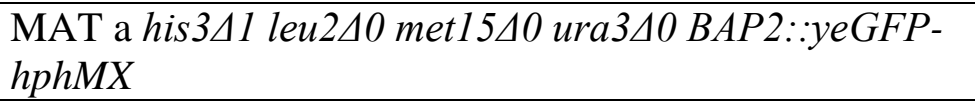 & This study \\
\hline
\end{tabular}




\begin{tabular}{|c|c|c|}
\hline $\begin{array}{l}\text { bull } \triangle B A P 2- \\
\text { yeGFP }\end{array}$ &  & This study \\
\hline
\end{tabular}

ST2. Plasmids.

\begin{tabular}{|l|l|l|}
\hline Plasmid & Description & Source \\
\hline YCplac33 & $\begin{array}{l}\text { Yeast centromeric shuttle vector with } A M P^{R} \text { and } \\
\text { URA3 markers }\end{array}$ & Lab stock \\
\hline YCplac33-BUL1 & $\begin{array}{l}\text { Yeast centromeric shuttle vector with } A M P^{R} \text { and } \\
U R A 3 \text { markers carrying } B U L 1\end{array}$ & This study \\
\hline YCplac33-BUL2 & $\begin{array}{l}\text { Yeast centromeric shuttle vector with } A M P^{R} \text { and } \\
\text { URA3 marker carrying BUL2 }\end{array}$ & This study \\
\hline YEplac181 & $\begin{array}{l}\text { Yeast episomal shuttle vector with } A M P^{R} \text { and } L E U 2 \\
\text { markers }\end{array}$ & Lab stock \\
\hline
\end{tabular}

\section{ST3. Primers.}

\begin{tabular}{|l|l|}
\hline Description & Sequence \\
\hline $\begin{array}{l}\text { Fwd Pr for } \\
\text { disruption of } B U L 1\end{array}$ & $\begin{array}{l}\text { 5'ACTGTTCGTGTGTGTCAACAGGTATATCGTACGCTAAATGGC } \\
\text { CAGCTAAGCTTCGTACG3' }\end{array}$ \\
\hline $\begin{array}{l}\text { Rev Pr for } \\
\text { disruption of } B U L 1\end{array}$ & $\begin{array}{l}\text { 5'TATAAGAAAAGTAACGAGAATTTTTTCTAATGTTTTTTTAGG } \\
\text { CCACTAGTGGATCTG3' }\end{array}$ \\
\hline $\begin{array}{l}\text { Fwd Pr for } \\
\text { confirmation of } \\
\text { disruption of } B U L 1\end{array}$ & 5'AGCGGGCCGTGACCCGCTCTCAGAGGCTAT3' \\
\hline $\begin{array}{l}\text { Fwd Pr for cloning } \\
\text { of } B U L 1\end{array}$ & $\begin{array}{l}\text { 5'TCTAGAGTCGACGTGTTCGTGTTGGAGCCTTCCTCTGGAGCA } \\
\text { TCGCCACCAG3' }\end{array}$ \\
\hline $\begin{array}{l}\text { Rev Pr for cloning } \\
\text { of } B U L 1\end{array}$ & $\begin{array}{l}\text { 5'GAGCTCGAATTCAGGACGCGATAAATACCTTCATCTATAAGC } \\
\text { 3' }\end{array}$ \\
\hline $\begin{array}{l}\text { Fwd Pr for cloning } \\
\text { of } B U L 2\end{array}$ & $\begin{array}{l}\text { 5'ATTACTGCAGGTCGACTACATATATTAGTACCATGACGTTAT } \\
\text { ATATATATCTGTGAAGAG3' }\end{array}$ \\
\hline $\begin{array}{l}\text { Rev Pr for cloning } \\
\text { of } B U L 2\end{array}$ & $\begin{array}{l}\text { 5'TAATGAGCTCGTCTCTCTTCTTCTTTAGTAATACCTGTGCCAT } \\
\text { GTGATAGCC3' }\end{array}$ \\
\hline $\begin{array}{l}\text { Fwd Pr for } \\
\text { disruption of } B A P 2\end{array}$ & $\begin{array}{l}\text { 5'CGATACTATCAATTTATTTAGCTTCAATAAAACTCAAGTGAT } \\
\text { TTAGAATGGCCAGCTGAAGCTTCGTACG3' }\end{array}$ \\
\hline $\begin{array}{l}\text { Rev Pr for } \\
\text { disruption of } B A P 2\end{array}$ & $\begin{array}{l}\text { 5'ATCAACCGGCTCTAATGGGAAGTGTCCAGACCTGAGTGGTGT } \\
\text { AGTAAGTTTAGGCCACTAGTGGATCTG3' }\end{array}$ \\
\hline $\begin{array}{l}\text { Fwd Pr for } \\
\text { confirmation of } \\
\text { disruption of } B A P 2\end{array}$ & \begin{tabular}{l} 
5'GATGAGCACATCTCCTCGTATATATACGTA' \\
\hline
\end{tabular} \\
\hline
\end{tabular}




\begin{tabular}{|c|c|}
\hline $\begin{array}{l}\text { Fwd Pr for } \\
\text { disruption of } B A P 3\end{array}$ & $\begin{array}{l}\text { 5'CCGGGCATTAAAAACTACGTTGTAATTAGCGTGGTTACTATT } \\
\text { GATGGATTGGCGAAGTAAGCCAGCTGAAGCTTCGTACG3' }\end{array}$ \\
\hline $\begin{array}{l}\text { Rev Pr for } \\
\text { disruption of } B A P 3\end{array}$ & $\begin{array}{l}\text { 5'TAGACAACGTTGAGTAAGGTGGTTCGGTCCGAACAACAGTTT } \\
\text { CATGCTTGTGTTACTTCGGGCCACTAGTGGATCTG3' }\end{array}$ \\
\hline $\begin{array}{l}\text { Fwd Pr for } \\
\text { confirmation of } \\
\text { disruption of } B A P 3\end{array}$ & 5'CAGTGGCAGTATGTAGTTTGTTCTTGCGTTTCCTTGTGGT3' \\
\hline $\begin{array}{l}\text { Fwd Pr for } \\
\text { disruption of TAT1 }\end{array}$ & $\begin{array}{l}\text { 5'GTAGCTACCTAATATAGTTTCTCGATAAAAAGGCGTAAAAAT } \\
\text { GGCCAGCTGAAGCTTCGTACG3' }\end{array}$ \\
\hline $\begin{array}{l}\text { Rev Pr for } \\
\text { disruption of TAT1 }\end{array}$ & $\begin{array}{l}\text { 5’AAGCCCGATGAAGCCAAGCGGAAAATGAATGGAATTGCTGT } \\
\text { TAGGCCACTAGTGGATCTG3' }\end{array}$ \\
\hline $\begin{array}{l}\text { Fwd Pr for } \\
\text { confirmation of } \\
\text { disruption of } T A T 1\end{array}$ & 5'GTTGGAGTAAAAAAGTATATAAGATGCTGT3' \\
\hline $\begin{array}{l}\text { Fwd Pr for } \\
\text { disruption of } T A T 2\end{array}$ & $\begin{array}{l}\text { 5'ACTGACTTTCTCAACACGTCGAGCACGACATTTCCCAAAAAT } \\
\text { AGCTGAAGAACATAAGCTAAGCAAATTACGCAACACACTCTC } \\
\text { ATATGGCCAGCTGAAGCTTCGTACG3' }\end{array}$ \\
\hline $\begin{array}{l}\text { Rev Pr for } \\
\text { disruption of } T A T 2\end{array}$ & $\begin{array}{l}\text { 5’TGGAATAATTAATTATGAAGGATGAGTACTAGAGAAGAGAA } \\
\text { AAAAAAATATTCTACAAAAATAAATTGAACTTGTTTCTTCGGT } \\
\text { ATTAGGCCACTAGTGGATCTG3' }\end{array}$ \\
\hline $\begin{array}{l}\text { Fwd Pr for } \\
\text { confirmation of } \\
\text { disruption of } T A T 2\end{array}$ & 5'GAGATTTCCCACCATTCACTCCAG3' \\
\hline $\begin{array}{l}\text { Fwd Pr for } \\
\text { disruption of } \\
\text { AGPl }\end{array}$ & $\begin{array}{l}\text { 5’TATTATGTAATCTTTATAGAAGAAGCACGCTAATATAGACAA } \\
\text { AGATAGCTTCGCACAATGGCCAGCTGAAGCTTCGTACG3' }\end{array}$ \\
\hline $\begin{array}{l}\text { Rev Pr for } \\
\text { disruption of } \\
A G P 1\end{array}$ & $\begin{array}{l}\text { 5'ACTACAAAACAGAAGTACAAAAATGAATAAATATAAAAGAA } \\
\text { GTAAATGCTTTTTTTTTTAGGCCACTAGTGGATCTG3' }\end{array}$ \\
\hline $\begin{array}{l}\text { Fwd Pr for } \\
\text { confirmation of } \\
\text { disruption of } \\
A G P 1\end{array}$ & 5'CCCGGGGGATTCCTGAAAGAGCGCCTTTACCTCAACCTAC3' \\
\hline $\begin{array}{l}\text { Fwd Pr for } \\
\text { disruption of } \\
\text { GNP1 }\end{array}$ & $\begin{array}{l}\text { 5'AGAAAAAAAAACTATCGCTTTCTCAAGTAGCTTATATAATAT } \\
\text { CAAATATTGCACATTATGGCCAGCTGAAGCTTCGTACG3' }\end{array}$ \\
\hline $\begin{array}{l}\text { Rev Pr for } \\
\text { disruption of } \\
G N P 1\end{array}$ & $\begin{array}{l}\text { 5'GCTAATTGAATTGTTTCTTCAAGTTTTTTTTTTTTTTTTTGAAT } \\
\text { CGTGATTTCTGCTTTAGGCCACTAGTGGATCTG3' }\end{array}$ \\
\hline $\begin{array}{l}\text { Fwd Pr for } \\
\text { confirmation of } \\
\text { disruption of } \\
G N P 1\end{array}$ & 5'GGGGATCCCTTGACCTCTGGCTTAGCTTCTTG3' \\
\hline $\begin{array}{l}\text { Fwd Pr for } \mathrm{C} \\
\text { terminal GFP } \\
\text { tagging of } B A P 2\end{array}$ & $\begin{array}{l}\text { 5'GAATATGTCTTTGATGAGAAAAGCTTATCATTTCTGGTGTGG } \\
\text { AGCAGGTGCTGGTGCTGGTGCTG3' }\end{array}$ \\
\hline
\end{tabular}




\begin{tabular}{|l|l|}
\hline $\begin{array}{l}\text { Rev Pr for C } \\
\text { terminal GFP } \\
\text { tagging of } B A P 2\end{array}$ & $\begin{array}{l}\text { 5'AATGGGAAGTGTCCAGACCTGAGTGGTGTAGTTAAGTTTATA } \\
\text { GTCCTGTCGGGTTTCGCCACC3' }\end{array}$ \\
\hline $\begin{array}{l}\text { Fwd Pr for } \\
\text { confirmation of C } \\
\text { terminal GFP } \\
\text { tagging of } B A P 2\end{array}$ & 5'ATGATCCAGAGCTAATGAGACAAGAGGACG3' \\
\hline $\begin{array}{l}\text { Fwd Pr for C } \\
\text { terminal GFP } \\
\text { tagging of } B A P 3\end{array}$ & $\begin{array}{l}\text { 5'AGAAAGGTTAAAGAACTCTTCAATATTCGTCAGAGTCTACAA } \\
\text { ATTGTGTGGAGCAGGTGCTGGTGCTGGTGCTG3' }\end{array}$ \\
\hline $\begin{array}{l}\text { Rev Pr } \mathrm{C} \\
\text { terminal GFP } \\
\text { tagging of } B A P 3\end{array}$ & 5'GTTGAGTAAGGTGGTTCGGTCCGAACAACAGTTTCATGCTTG \\
\hline $\begin{array}{l}\text { Fwd Pr for } \\
\text { confirmation of C } \\
\text { terminal GFP } \\
\text { tagging of } B A P 3\end{array}$ & 5'CGAGAGTTATCTGGCTGCCCCGCTATGGAT3' \\
\hline $\begin{array}{l}\text { Primer for } \\
\text { confirmation of } \\
\text { tagging by yeGFP }\end{array}$ & 5' TTATTTGTACAATTCATCCATACCATGGGTAATACC3' \\
\hline $\begin{array}{l}\text { Primer for } \\
\text { confirmation of } \\
\text { tagging by EGFP }\end{array}$ & 5'CCGCCGAATCCTTACTTGTACAGCTCGTCCATGCCG3' \\
\hline
\end{tabular}




\section{References}

Abe, F., and H. Iida. 2003. Pressure induced differential regulation of the two tryptophan permeases Tat 1 and Tat 2 by ubiquitin ligase Rsp5 and its binding proteins, Bul1 and Bul2. Mol. Cell. Biol. 23:7566-7584.

Alvers, A.L., L.K. Fishwick, M.S. Wood, D. Hu, H.S. Chung, W.A. Dunn Jr and J.P. Aris. 2009. Autophagy and amino acid homeostasis are required for chronological longevity in Saccharomyces cerevisiae. Aging cell. 8(4):353-369.

Ananieva, E.A., J.D. Powell, and S.M. Hutson. 2016. Leucine Metabolism in T Cell Activation: mTOR Signaling and Beyond. Adv Nutr. 7(4):798S-805S.

Becuwe, M., N. Vieira, D. Lara, J. Gomes-Rezende, C. Soares-Cunha, M. Casal, R. HaguenauerTsapis, O. Vincent, S. Paiva, and Léon S. 2012a. A molecular switch on an arrestin-like protein relays glucose signaling to transporter endocytosis. J. Cell Biol. 196:247-259.

Belgareh-Touzé, N., S. Léon, Z. Erpapazoglou, M. Stawiecka-Mirota, D. Urban-Grimal, and R. Haguenauer-Tsapis. 2008. Versatile role of the yeast ubiquitin ligase Rsp5 in intracellular trafficking. Biochem Soc Trans. 36(5):791-796.

Bonfils, G., M. Jaquenoud, S. Bontron , C. Ostrowicz, C. Ungermann, and C. De Virgilio. 2012. Leucyl-tRNA synthetase controls TORC1 via the EGO complex. Mol Cell. 46:105-110.

Cohen, R., and D. Engelberg. 2007. Commonly used Saccharomyces cerevisiae strains (e.g. BY4741, W303) are growth sensitive on synthetic complete medium due to poor leucine uptake. FEMS Microbiol Lett. 273(2):239-243.

Crapeau, M., A. Merhi, and B. André. 2014. Stress conditions promote yeast Gap1 permease ubiquitylation and down-regulation via the arrestin-like Bul and Aly proteins. J. Biol. Chem. 289:22103-22116.

Crozier, S.J., S.R. Kimball, S.W. Emmert, J.C. Anthony, and L.S. Jefferson. 2005. Oral leucine administration stimulates protein synthesis in rat skeletal muscle. J. Nutr. 135:376-382.

Didion, T., B. Regenberg, M.U. Jørgensen, M.C. Kielland-Brandt, and H.A. Andersen. 1998. The Permease Homologue Ssylp Controls the Expression of Amino Acid and Peptide Transporter Genes in Saccharomyces Cerevisiae. Mol Microbiol. 27(3):643-50.

Forsberg, H., M. Hammar, C. Andreasson, A. Moliner, and P.O. Ljungdahl. 2001. Suppressors of ssy1 and ptr3 null mutations define novel amino acid sensor-independent genes in Saccharomyces cerevisiae. Genetics. 158:973-88.

Ghaddar, K., A. Merhi, E. Saliba, E.M. Krammer, M. Prévost, and B. Andre. 2014. Substrate Induced Ubiquitylation and Endocytosis of Yeast Amino Acid Permeases. Mol Cell Biol. 34(24):4447-4463. 
Gonzalez, A., and M.N. Hall. 2017. Nutrient sensing and TOR signaling in yeast and mammals. EMBO J. 36(4):397-408.

Grauslund, M., T. Didion, M.C. Kielland-Brandt, and H.A. Andersen. 1995. BAP2, a Gene Encoding a Permease for Branched-Chain Amino Acids in Saccharomyces Cerevisiae. Biochim Biophys Acta. 1269(3):275-80.

Han, J.M., S.J. Jeong, M.C. Park, G. Kim, N.H. Kwon, H.K. Kim, S.H. Ha, S.H. Ryu, and S. Kim. 2012. Leucyl-tRNA synthetase is an intracellular leucine sensor for the mTORC1-signaling pathway. Cell. 149:410-424.

Hatakeyama, R., M. Kamiya, T. Takahara, and T. Maeda. 2010. Endocytosis of the aspartic acid/glutamic acid transporter Dip5 is triggered by substrate-dependent recruitment of the Rsp5 ubiquitin ligase via the arrestin-like protein Aly2. Mol. Cell. Biol. 30:5598-5607.

Hettema, E.H., J. Valdez-Taubas, and H.R.B Pelham. 2004. Bsd2 binds the ubiquitin ligase Rsp5 and mediates the ubiquitination of transmembrane proteins. EMBO J. 23(6):1279-1288.

Hovsepian, J., Q. Defenouillere, V. Albanese, L. Vachova, C. Garcia, Z. Palkova, and S. Leon. 2017. Multilevel regulation of an alpha-arrestin by glucose depletion controls hexose transporter endocytosis. J. Cell Biol. 216:1811-1831.

Hovsepian, J., V. Albanese, M. Becuwe, V. Ivashov, D. Teis, and S. Leon. 2018. The yeast arrestin-related protein Bul1 is a novel actor of glucose-induced endocytosis. Mol. Biol. Cell. 29:1012-1020.

Kwan, E.X., E. Foss, L. Kruglyak, and A. Bedalov. 2011. Natural Polymorphism in BUL2 Links Cellular Amino Acid Availability With Chronological Aging and Telomere Maintenance in Yeast. Plos Genet. 7(8):e1002250.

Lauwers, E., C. Jacob, and B. André. 2009. K63-linked ubiquitin chains as a specific signal for protein sorting into the multivesicular body pathway. J Cell Biol. 185(3):493-502.

Leon, S., and R. Haguenauer-Tsapis. 2009. Ubiquitin Ligase Adaptors: Regulators of Ubiquitylation and Endocytosis of Plasma Membrane Proteins. Exp Cell Res. 315(9):1574-83.

Lin, C.H., J.A. MacGurn, T. Chu, C.J Stefan, and S.D. Emr. 2008. Arrestin related ubiquitin ligase adaptors regulate endocytosis and protein turnover at the cell surface. Cell. 135:714-725.

Ljungdahl, P.O., and B. Daignan-Fornier. 2012. Regulation of Amino Acid, Nucleotide, and Phosphate Metabolism in Saccharomyces cerevisiae. Genetics. 190:885-929.

Merhi, A., and B. Andre. 2012. Internal amino acids promote Gap1 permease ubiquitylation via TORC1/Npr1/14-3-3-dependent control of the Bul arrestin-like adaptors. Mol. Cell. Biol. 32: 4510-4522. 
Nikko, E., J.A. Sullivan, and H.R. Pelham. 2008. Arrestin-like proteins mediate ubiquitination and endocytosis of the yeast metal transporter Smf1. EMBO Rep. 9:1216-1221.

Nikko, E., and H.R. Pelham. 2009. Arrestin-mediated endocytosis of yeast plasma membrane transporters. Traffic. 10:1856-1867.

O’Donnell, A.F., A. Apffel, R.G. Gardner, and M.S. Cyert. 2010. Alpha-arrestins Aly1 and Aly2 regulate intracellular trafficking in response to nutrient signaling. Mol. Biol. Cell. 21:3552-3566.

O’Donnell, A.F., and M.C. Schmidt. 2019. AMPK-Mediated Regulation of Alpha-Arrestins and Protein Trafficking. Int J Mol Sci. 20(3):515.

Peter, G.J., L. Düring, and A. Ahmed. 2006. Carbon Catabolite Repression Regulates Amino Acid Permeases in Saccharomyces cerevisiae via the TOR Signaling Pathway. J Biol Chem. 281(9):5546-52.

Renguet, E., A. Ginion, R. Gélinas, L. Bultot, J. Auquier, I.R. Frayne, C. Daneault, J.L. Vanoverschelde, C. Des Rosiers, L. Hue, S. Horman, C. Beauloye, and L. Bertrand. 2017. Metabolism and acetylation contribute to leucine-mediated inhibition of cardiac glucose uptake. Am J Physiol Heart Circ Physiol. 313(2):H432-H445.

Rotin, D., O. Staub, and R. Haguenauer-Tsapis. 2000. Ubiquitination and Endocytosis of Plasma Membrane Proteins: Role of Nedd4/Rsp5p Family of Ubiquitin-Protein Ligases. J Membr Biol. 176(1):1-17.

Savocco, J., S. Nootens, W. Afokpa, M. Bausart, X. Chen, J. Villers, H. Renard, M. Prévost, R. Wattiez, and P. Morsomme. 2019. Yeast $\alpha$-Arrestin Art2 Is the Key Regulator of UbiquitylationDependent Endocytosis of Plasma Membrane Vitamin B1 Transporters. Plos Biol. 17(10):e3000512.

Stipanuk, M.H. 2007. Leucine and protein synthesis: mTOR and beyond. Nutr. Rev. 65:122-129.

Suryawan, A., R.A. Orellana, M.L. Fiorotto, and T.A. Davis. 2011. Leucine acts as a nutrient signal to stimulate protein synthesis in neonatal pigs. J Anim Sci. 89(7):2004-2016.

Suzuki, A., T. Mochizuki, S. Uemura, T. Hiraki, and F. Abe. 2013. Pressure-induced endocytic degradation of the Saccharomyces cerevisiae low-affinity tryptophan permease Tat1 is mediated by Rsp5 ubiquitin ligase and functionally redundant PPxY motif proteins. Eukaryot. Cell. 12:990-997.

Talaia, G., C. Gournas, E. Saliba, C. Barata-Antunes, M. Casal, B. Andre, G. Diallinas, and S. Paiva. 2017. The alpha-Arrestin Bul1p Mediates Lactate Transporter Endocytosis in Response to Alkalinization and Distinct Physiological Signals. J. Mol. Biol. 429:3678-3695. 
Tanahashi, R., Matsushita, T., Nishimura, A. and Takagi, H. 2021. Downregulation of the broadspecificity amino acid permease Agp1 mediated by the ubiquitin ligase Rsp5 and the arrestin-like protein Bul1 in yeast. Biosci Biotechnol Biochem. 85:1266-1274.

Umebayashi. K and Nakano. A. 2003. Ergosterol is required for targeting of tryptophan permease to the yeast plasma membrane. J. Cell Biol. 161:1117-1131.

Xiao, F., C. Wang, H. Yin, J. Yu, S. Chen, J. Fang, and F. Guo. 2016. Leucine deprivation inhibits proliferation and induces apoptosis of human breast cancer cells via fatty acid synthase. Oncotarget. 7(39):63679-63689.

Yashiroda, H., D. Kaida, A. Toh-e, and Y. Kikuchi. 1998. The PY-motif of Bul1 protein is essential for growth of Saccharomyces cerevisiae under various stress conditions. Gene. 225:3946.

Zhao, Y., J.A. Macgurn, M. Liu, and S.D. Emr. 2013. The ART-Rsp5 ubiquitin ligase network comprises a plasma membrane quality control system that protects yeast cells from proteotoxic stress. eLife. 2:e0459.

\section{Acknowledgements}

We are very grateful to Department of Science and Technology, INDIA, for funding the project (P13DST024).

\section{Conflict of interest}

The authors declare no conflict of interest.

\section{Author contribution}

MS and PJB conceived ideas and designed the experiments. MS performed the experiments. MS and PJB analyzed the results. MS and PJB wrote the manuscript.

\section{Data availability statement}

Data supporting the findings of this study are available, upon request. 